\title{
Propriétés des substituts de présure fabriqués à partir de Mucor miehei
}

\author{
par \\ A. REPS, S. POZNANSKI, A. BABUCHOWSKI \\ et L. JEDRYCHOWSKI \\ Institut du Génie et de la Biotechnologie des Produits Alimentaires \\ de l'Université de Technologie Agricole Olsztyn (Pologne)
}

\section{INTRODUCTION}

Les substituts les plus connus de la présure qui sont fabriqués à partir de moisissures, sont les suivants : la préparation "Meito ", produite au Japon à partir de Mucor pusillus, et la préparation "Suparen ", fabriquée aux Etats-Unis à partir d'Endothia parasitica. De l'avis de plusieurs auteurs, les préparations citées ne correspondent pas pleinement, par leurs propriétés, à la présure de veau.

Bien que l'utilisation de ces préparations soit bien répandue dans la fabrication des fromages, des recherches sont continuées par plusieurs laboratoires de Sociétés diverses afin d'obtenir de nouveaux substituts ayant des propriétés analogues à celles de la présure.

$\mathrm{Au}$ cours des dernières années, plusieurs renseignements ont été publiés concernant des préparations coagulantes obtenues à partir de la moisissure Mucor miehei. Selon Koning et Draaisma [6], la production des substituts de présure à partir de la moisissure Mucor miehei a été réalisée par trois sociétés :

- Société Rapidase (France), la préparation «Fromase » en poudre et en solution ;

- Novo Industri A/S (Danemark), la préparation « Rennilase » en poudre et en solution ;

- Marschall Division, Miles Laboratories Inc. (Etats-Unis), la préparation « Marzyme » en solution.

Ce rapport présente les résultats des études effectuées sur des propriétés physico-chimiques des préparations mentionnées, obtenues à partir de la moisissure Mucor miehei. L'attention est surtout prêtée aux propriétés qui jouent un rôle important dans la fabrication des fromages. 


\section{PROCEDE EXPERIMENTAL}

Dans nos études, on a comparé les propriétés des préparations en poudre, c'est-à-dire celles de " Fromase " et de "Rennilase ", à l'activité coagulante de 1:100 000, ainsi que la préparation « Marzyme ", en solution, à l'activité de 1:24 000. Comme standard, on a choisi la solution de présure de veau, à l'activité coagulante de 1:16 000. Le lait cru, écrémé, servait de substrat. A chaque échantillon analysé, on ajoutait une quantité de préparation ou de présure qui coagulait le lait en vrac, cru, écrémé en $30 \mathrm{mn}$. La température optimum de coagulation du lait, ainsi que le temps de la floculation étaient déterminés, pour les préparations analysées, par les méthodes que nous avons décrites dans une publication précédente [7].

La fermeté du caillé était mesurée à l'aide de l'appareil construit par Eisele et al. [2]. La détermination était faite sur le lait cru, en vrac, écrémé, pasteurisé à $75^{\circ} \mathrm{C}$ pendant $16 \mathrm{~s}$, ainsi que sur le même lait pasteurisé, additionné de 0,02 p.100 de chlorure de calcium.

\section{RESULTATS ET DISCUSSION}

La température optimale de la coagulation du lait était plus élevée pour les préparations de "Fromase ", "Rennilase " et " Marzyme " que pour la présure $\left(40-44^{\circ} \mathrm{C}\right)$; elle est de l'ordre de 58 à $62^{\circ} \mathrm{C}$. Elle est aussi plus élevée en comparaison avec des préparations que nous avons examinées précédemment, les préparations « Meito » $\left(42-45^{\circ} \mathrm{C}\right.$ ) et "Suparen » $\left(42^{\circ} \mathrm{C}\right)$ [9]. Sous ce rapport aucunes différences n'ont été constatées pour les trois préparations examinées actuellement (fig. 1). Des résultats semblables ont été obtenus, concernant la température optimale, par Prins et Nielsen [8], Ibrahim et al. [5] ainsi que Houins [4]. Par contre, Hamdy et Edelstein [3] ont trouvé une température optimale de coagulation de $42^{\circ} \mathrm{C}$ pour la préparation " Rennilase ». Il faut particulièrement souligner la différence appréciable entre température d'inactivation de la présure et celles des préparations examinées, qui dépassait la valeur de $20^{\circ} \mathrm{C}$, ce qui est sans doute important pour l'utilisation de ces préparations dans la fabrication des fromages chauffés à une température élevée où se produit l'inactivation des enzymes de la présure. La température d'inactivation est de l'ordre de $53^{\circ} \mathrm{C}$ pour la préparation « Meito », $45^{\circ} \mathrm{C}$ pour la préparation «Suparen » et $73^{\circ} \mathrm{C}$ pour la " Fromase ».

Aucunes différences du temps de floculation n'ont été constatées entre les trois préparations examinées et la présure, en fonction des variations d'acidité de lait, pour des valeurs du $\mathrm{pH}$ entre 6,7 et 5,1 . Pour les trois préparations examinées, les mêmes modifications des courbes de temps de floculation ont été observées que pour celles de la présure. C'est un grand avantage pratique de ces préparations, 


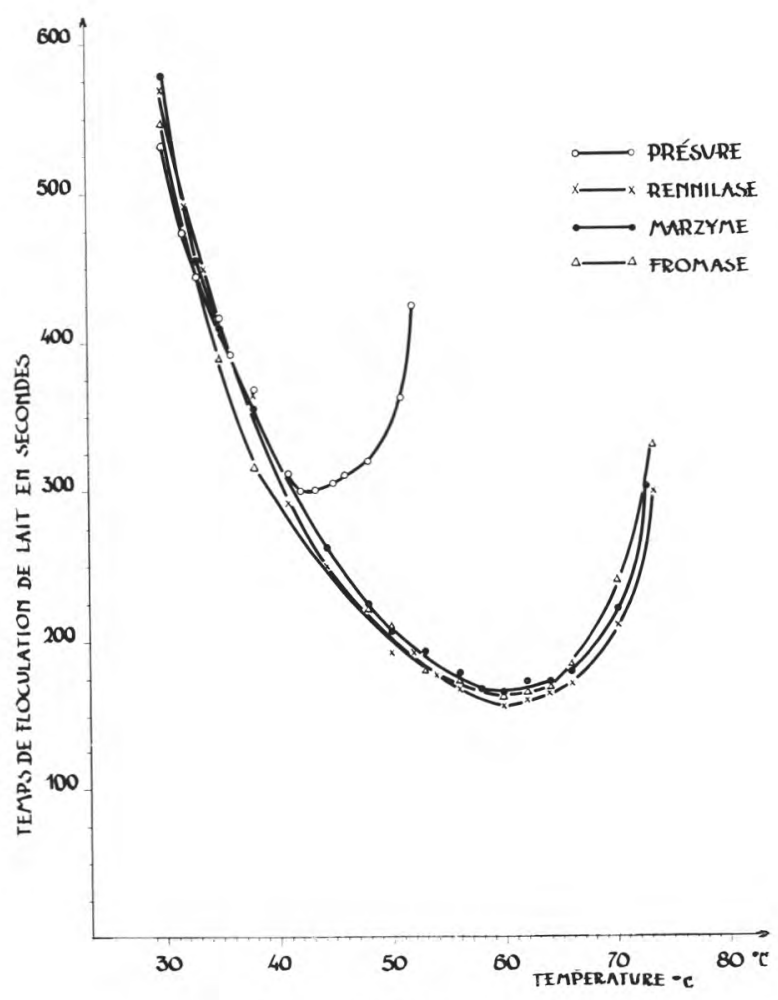

fig. 1

Temps de floculation du lait au cours de l'utilisation des préparations enzymatiques en fonction de la température.

parce que les préparations "Meito " et "Suparen », étudiées précédemment, se montraient plus sensibles aux changements du taux d'acidité du lait [9], ce qui rend le standard de fromage plus difficile à maintenir.

L'activité de coagulation du lait des préparations examinées dépend, plus que pour celle de la présure, des quantités de chlorure de calcium, ajoutées au lait, bien qu'on n'ait pas constaté de différences entre les trois préparations. Cependant en ajoutant 0,02 p. 100 de chlorure de calcium, les différences sont petites, l'activité augmente de 35,5 p. 100 , en comparaison avec 35 p. 100 pour la présure. En ajoutant 0,05 p. 100 de chlorure de calcium, l'augmentation d'activité est de 62 à 64 p. 100, en comparaison avec 52,5 p. 100 pour la présure. L'addition de 0,1 p. 100 fait augmenter l'activité des préparations de 80 p. 100 et celle de la présure de 71 p. 100, et celle de 0,15 p. 100, 
la fait augmenter de 85 p. 100 pour les préparations et 77 p. 100 pour la présure. Alais et Lagrange ont obtenu [1] des résultats semblables en analysant la préparation "Rennilase ». Des propriétés analogues étaient démontrées pour les préparations « Meito » et "Suparen » [9]. Pour augmenter la coagulabilité du lait de fromage on y a ajouté 0,02 p.100 de chlorure de calcium, les différences constatées étant ainsi pratiquement peu importantes (fig. 2).

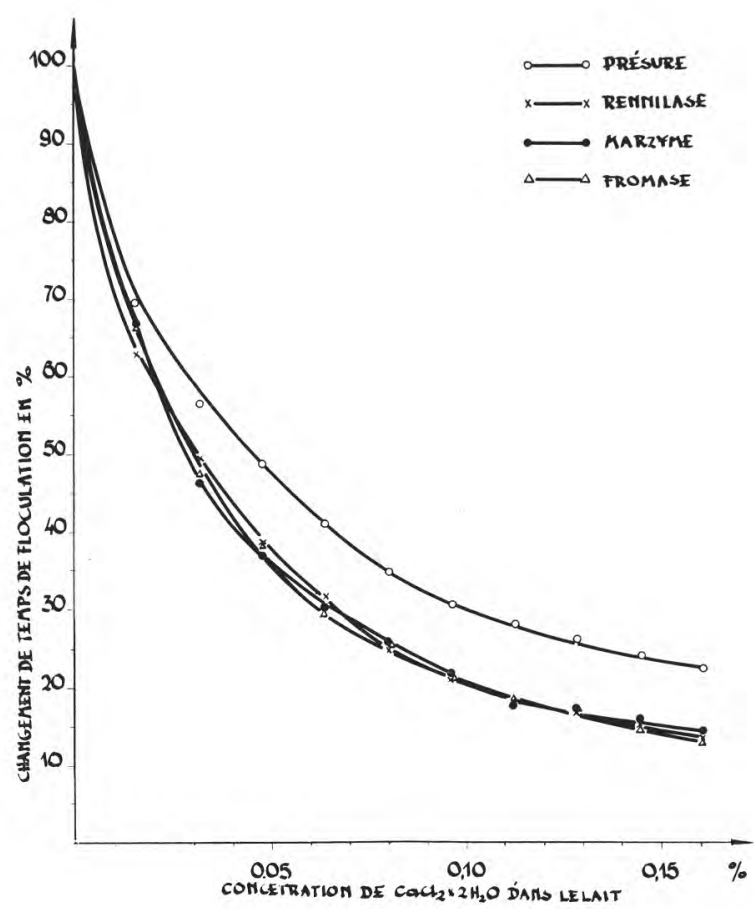

fig. 2

Influence de de l'addition de $\mathrm{CaCl}_{2}+2 \mathrm{H}_{2} \mathrm{O}$ dans le lait sur le temps de floculation au cours de l'utilisation des préparations enzymatiques.

Un effet évident sur le changement d'activité a été exercé par le chlorure de sodium ajouté au lait. Par rapport à la présure, qui est bien sensible à la présence de chlorure de sodium, les préparations microbiologiques démontrent l'activité augmentée de coagulation jusqu'à la concentration de 0,5 p. 100. Puis, l'augmentation continuée du chlorure de sodium fait surtout diminuer l'activité de coagulation 
de la préparation "Marzyme » et ensuite celle de la "Rennilase » (fig. 3). Sous ce rapport, "Rennilase » et "Marzyme " diffèrent plus de la présure, que les préparations " Meito » et "Suparen », analysées précédemment, tout en ressemblant à la préparation japonaise «Milcozyme »[9].

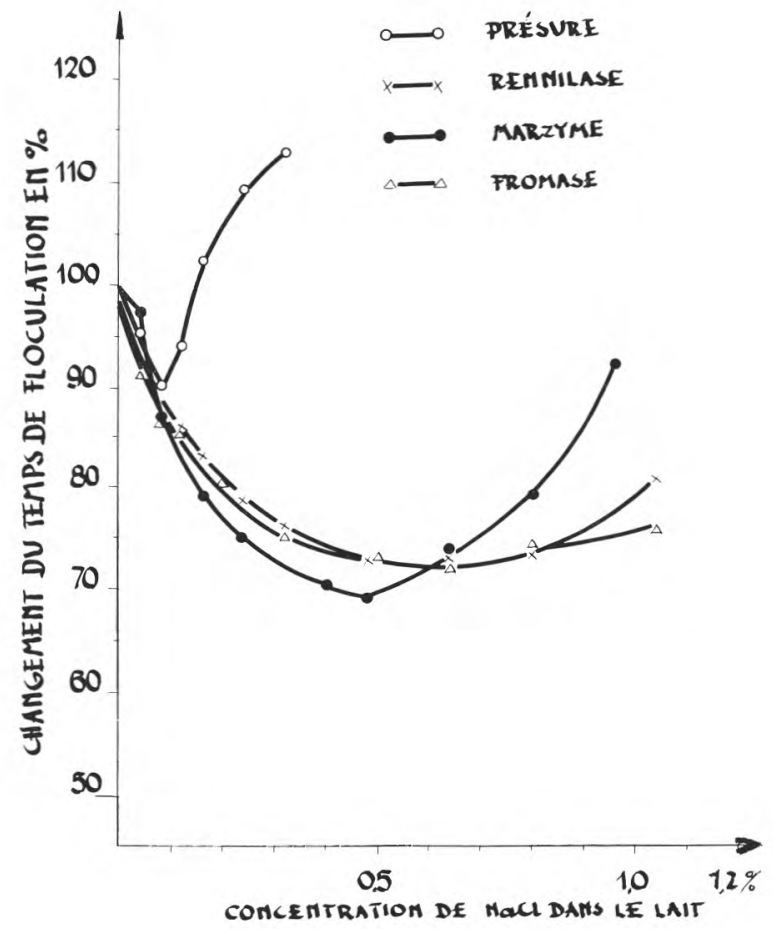

fig. 3

Influence de l'addition de $\mathrm{NaCl}$ dans le lait sur le temps de floculation au cours de l'utilisation des préparations enzymatiques.

Dans le cas de la coagulation par la présure, la valeur du produit de la quantité de présure par le temps de floculation du lait se maintient constante. Les préparations « Fromase » et " Marzyme » ne diffèrent que bien peu de la présure, tandis que la "Rennilase " montre une différenciation plus évidente, on a aussi trouvé possible de mélanger la présure et les préparations analysées en proportion variée pour les employer à coaguler le lait. Ajoutées à la solution de présure, 
les préparations examinées faisaient diminuer le temps de floculation du lait proportionnellement à la quantité introduite d'une préparation. En utilisant un mélange de présure et des préparations examinées pour un $\mathrm{pH}$ du lait variant de 6,7 à 6,2 (par intervalle de 0,1), les changements d'activité sont les mêmes que ceux de la présure.

La propriété la plus essentielle d'une préparation coagulante qui décide de son utilité dans la fabrication de fromage, c'est le caractère du caillé obtenu. En pratique, cette propriété est définie par la fermeté du caillé.

En employant la préparation "Fromase », ou son mélange avec la présure, dans le lait cru, on a obtenu les mêmes courbes de fermeté du caillé que dans le cas de la présure seule. Par contre, la fermeté du caillé obtenu avec des préparations " Rennilase 》 et "Marzyme " était de 30 p. 100 plus faible (fig. 4).

La fermeté du caillé obtenu avec la préparation "Fromase » à partir du lait pasteurisé, était la même que celle du caillé de la présure. Elle était un peu plus faible pour la préparation " Marzyme » et de $12 \mathrm{p} .100$ inférieure pour la préparation "Rennilase » (fig. 5). La fermeté des caillés qui étaient obtenus à partir du mélange de ces préparations avec la présure (1:1), déterminée de manière analogue, était de 30 p. 100 plus faible.

En pratique, pendant la fabrication des fromages à partir du lait pasteurisé, on ajoute 0,02 p. 100 de chlorure de calcium. On en a alors étudié l'influence sur la fermeté des caillés, qui étaient obtenus avec des préparations examinées et leurs mélanges avec la présure [9].

La fermeté des caillés qui étaient obtenus par la "Fromase " et le « Marzyme » était de 6,5 p. 100 plus faible qu'en cas de caillé de présure (fig. 6).

Le mélange " présure - Fromase » donnait le caillé ayant une fermeté analogue à celle obtenue à l'aide de la présure seule. Avec le mélange " présure - Rennilase » la fermeté du caillé obtenu était de 8 p. 100 plus faible, et, avec le mélange " Marzyme - présure ", elle était de 10 p. 100 plus faible, en comparaison avec celle du caillé de présure. Ces résultats laissent constater que la préparation "Fromase ", seule ou mélangée avec la présure en proportion de 1:1, produit un caillé, dont la fermeté ne diffère que bien peu de celle du caillé de présure.

On a aussi déterminé l'effet du chlorure de calcium, ajouté au lait pasteurisé, sur la durée de la coagulation du lait traité par les préparations examinées. En tous cas, l'addition de 0,2 p. 100 de $\mathrm{CaCl}_{2}$ faisait légèrement réduire la durée de la coagulation de la manière suivante (fig. 7) : la présure-1ait cru 15, lait pasteurisé avec 0,02 p. 100 de $\mathrm{CaCl}_{2} 14$, «Fromase »- lait cru 13, lait pasteurisé 12, "Rennilase »lait cru et pasteurisé $12 \mathrm{mn}$, « Marzyme »- lait cru et pasteurisé $14 \mathrm{mn}$. Cependant, une certaine réduction de la "phase enzymatique " a été observée dans le lait pasteurisé contenant 0,02 p. $100 \mathrm{de} \mathrm{CaCl}_{2}$, qui 


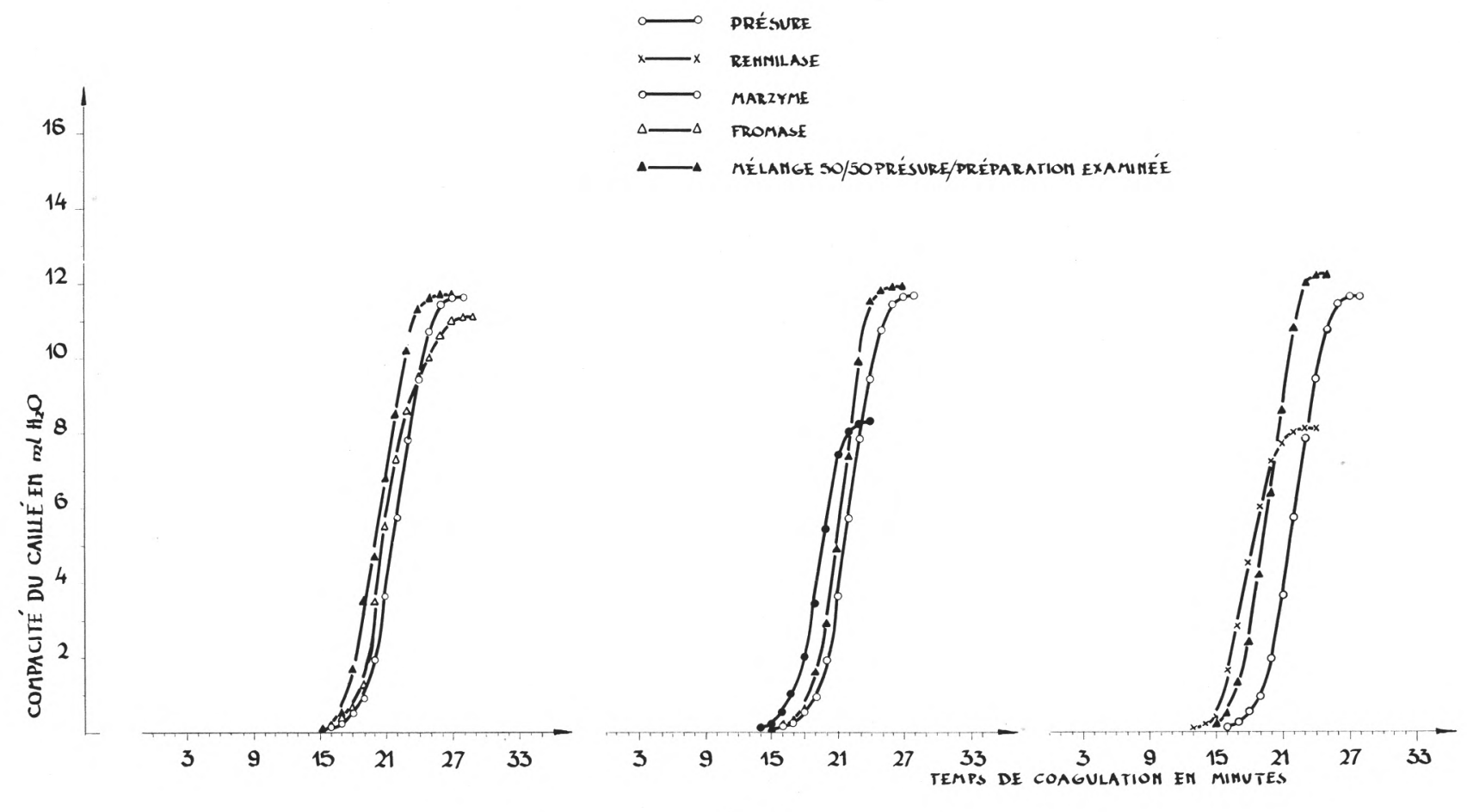

fig. 4

Compacité du caillé de lait non pasteurisé coagulé par les préparations examinées ou leur mélange avec la présure. 

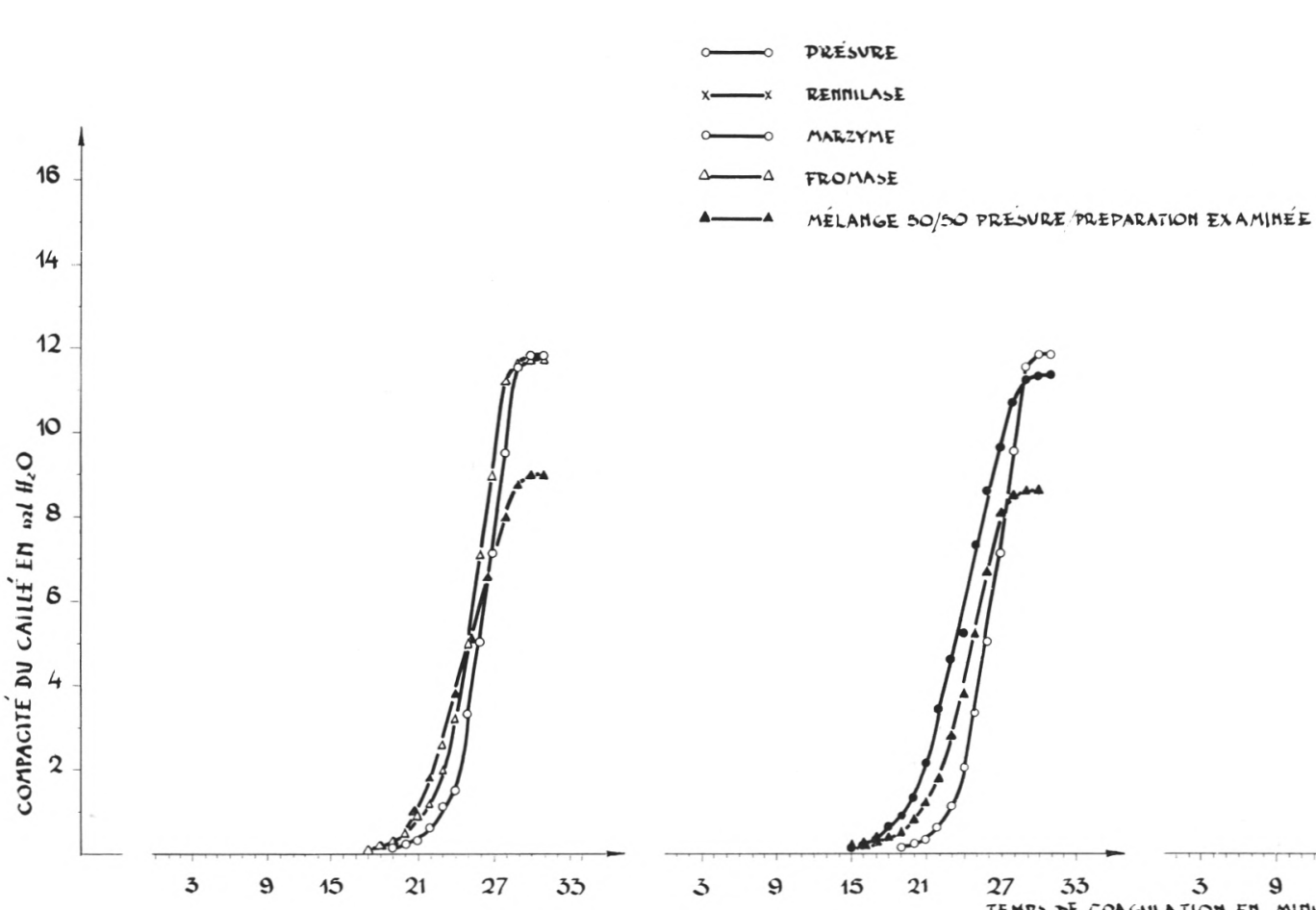

-A MELAMGE 5O/SO PRESVRE PREPARATLOH EX AMIMEE

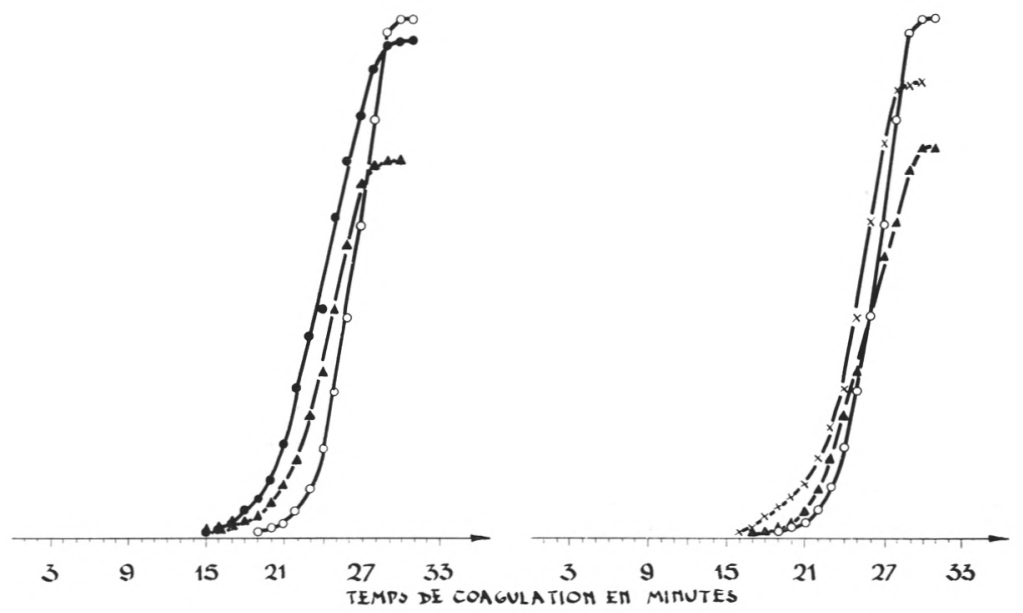

fig. 5

Compacité du caillé de lait pasteurisé coagulé par les préparations examinées ou leur mélange avec la présure. 

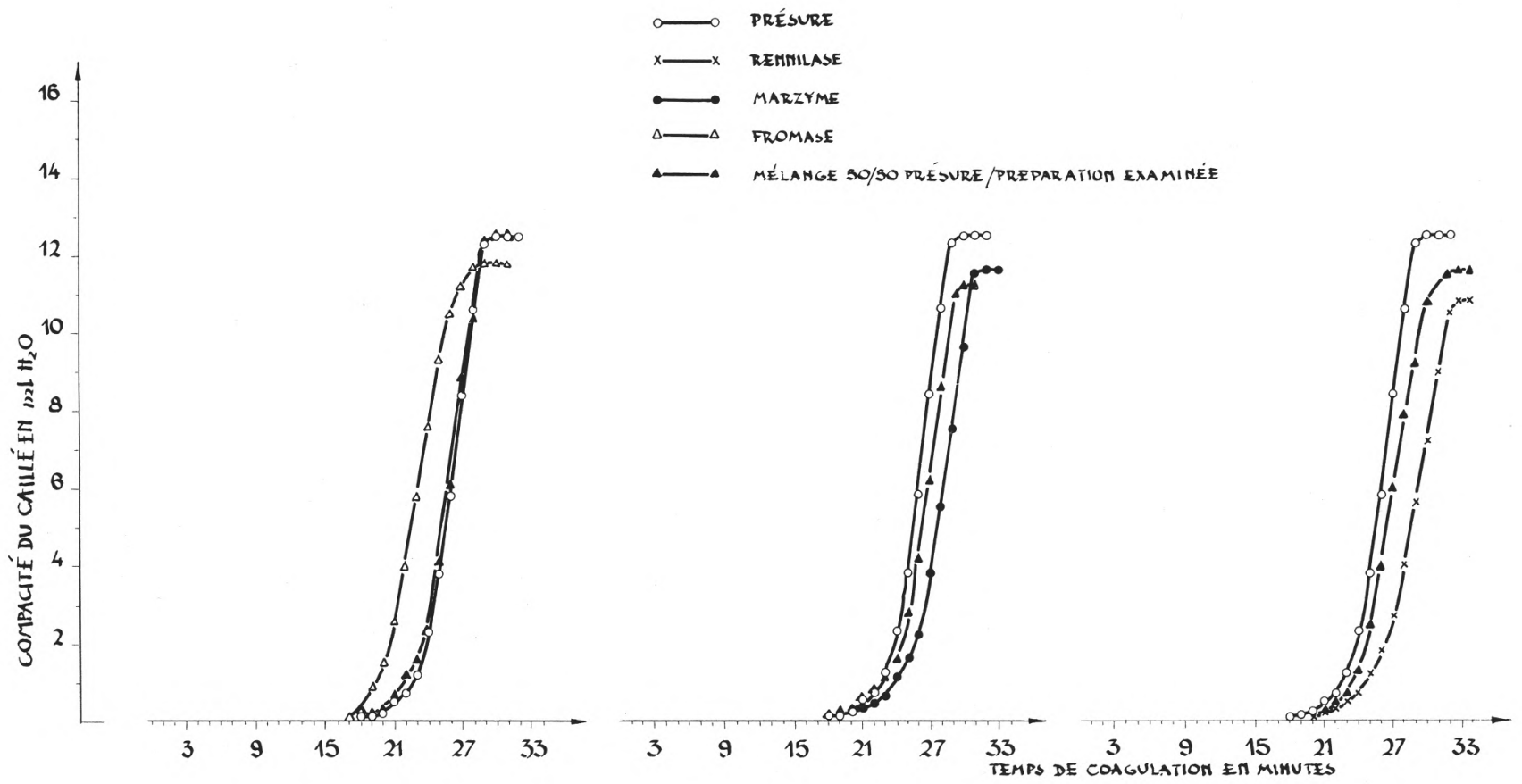

fig. 6

Compacité du caillé de lait pasteurisé $+0,02$ p. $100 \mathrm{CaCl}_{2}$ coagulé par les préparations examinées ou leur mélange avec la présure. 

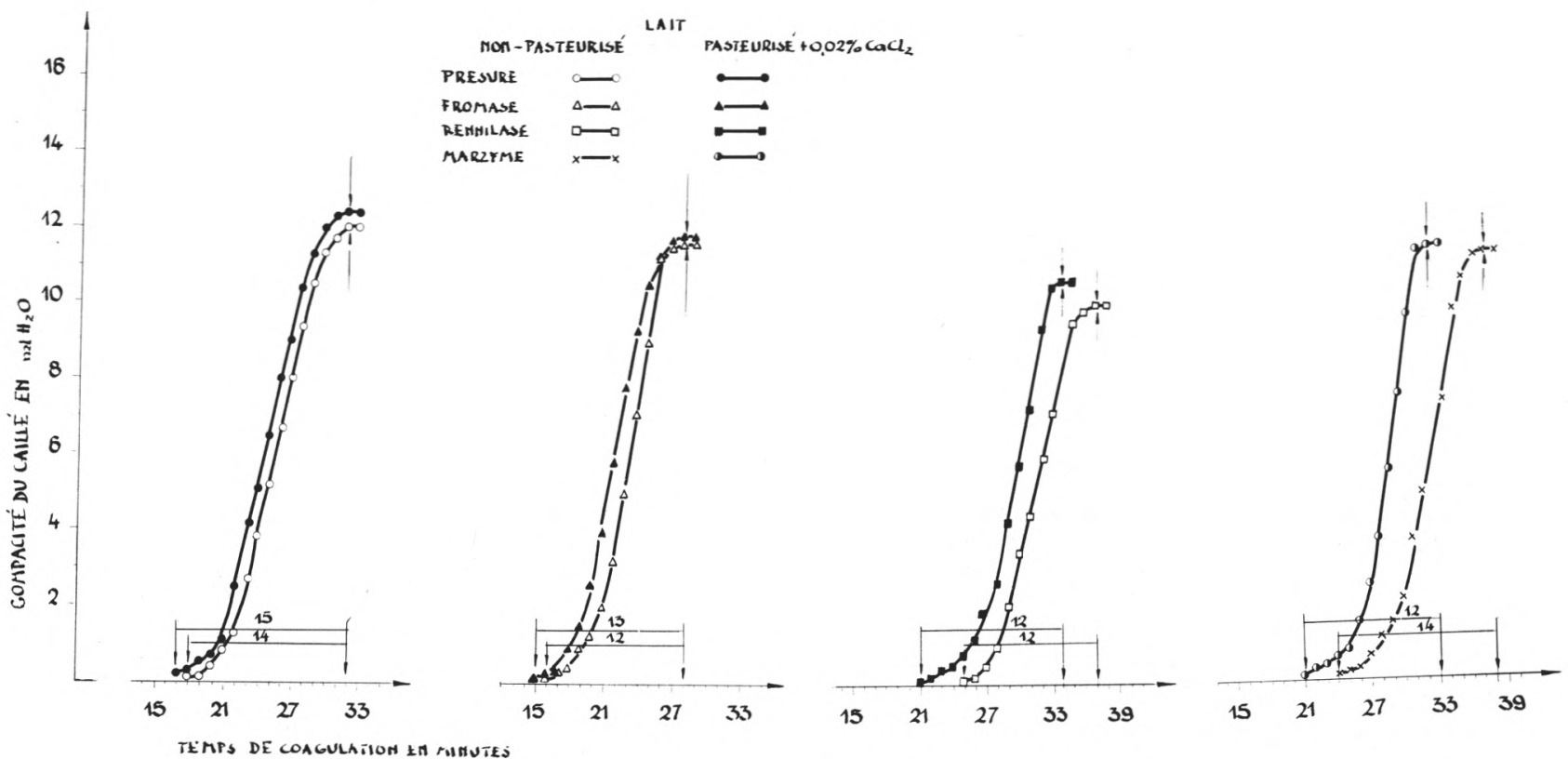

fig. 7

Influence de l'addition de $\mathrm{CaCl}_{2}$ dans le lait pasteurisé sur la durée de la phase de coagulation du lait en utilisant les préparations enzymatiques examinées. 
était additionné des préparations "Rennilase » et « Marzyme ". Pour la "Rennilase », la phase enzymatique était réduite de 25 à $21 \mathrm{mn}$, pour le "Marzyme " de 24 à $21 \mathrm{mn}$, le temps étant mesuré depuis le moment où la préparation avait été introduite dans le lait jusqu'à celui où l'apparition du caillé était signalée par l'appareil. On n'a observé, pourtant, aucune influence de $\mathrm{CaCl}_{2}$, ajouté au lait pasteurisé, sur la durée de la phase enzymatique du lait coagulé par la présure ou par la préparation «Fromase ».

Nos études ont démontré, que, sauf la température optimale de coagulation du lait et la température d'inactivation, les propriétés des préparations examinées se rapprochent plus de celles de la présure que de celles des préparations étudiées précédemment. De plus, les préparations examinées à présent ( "la Fromase », "la Rennilase » et " le Marzyme ») se distinguent par des propriétés qui paraissent plus désirables que celles des substituts de présure mieux connus jusqu'à présent.

\section{Ré s u m é}

On a étudié les propriétés physico-chimiques des préparations : "Fromase ", " Rennilase » et " Marzyme " qui sont produites à partir de la moisissure Mucor miehei. On a déterminé la température optimale de floculation du lait et la température d'inactivation d'un enzyme, la relation entre le temps de floculation et le $\mathrm{pH}$ du lait ainsi que des quantités de $\mathrm{CaCl}_{2}$ ajoutées au lait, de même que la relation entre le temps de floculation et la quantité des préparations ajoutée, ainsi que la proportion entre la préparation et la présure dans le mélange.

On a présenté des variations sur la fermeté du caillé de lait cru, de lait pasteurisé et de lait pasteurisé additionné de chlorure de calcium.

La température optimale de coagulation du lait, ainsi que la température d'inactivation des préparations examinées sont bien plus élevées que les valeurs correspondantes pour la présure.

Aucunes différences n'ont été constatées entre les propriétés des préparations examinées. Leurs propriétés, et surtout celles de la "Fromase ", sont bien comparables à celles de la présure.

\section{S u m m a r y}

In series of our experiments the basic physico-chemical properties of rennet substitutes of Mucor miehei, "Fromase», "Rennilase», "Marzyme» were analyzed. Optimum milk coagulation temperature, temperature of enzym inactivation, relationships between coagulation time and amount of added enzyme, coagulation time and amount of 
added $\mathrm{CaCl}_{2}$, coagulation time and $\mathrm{pH}$ of milk, coagulation time and amount of added pure preparations or mixtures with various ratios with rennet were determined.

The differences between hardness of coagulum from raw, pasteurized and nonpasteurized milk were find.

Optimum temperature of milk coagulation and temperature of enzymes inactivation for examined preparations were much higher than temperature of rennet inactivation. No significant differences were observed between properties of tested preparations. The properties of preparations especially «Fromase» are similar to rennet one.

Reçu pour publication en juin 1978.

\section{Bibliographie}

[1] Alais (C.), Lagrange (A.) (1972). - Etude biochimique d'une protéase coagulante produite par Mucor miehei. Le Lait, 517, 407.

[2] Eisele (M.), Babuchowski (K.), Budny (J.) (1962). - Próba analizy procesu podpuszczkowego scinania mleka metoda fizyczna. Zesz. Nauk. WSR, Olsztyn, 13, 237.

[3] Hamdy (A. D.), Edelsten (J.) (1970). - Some factors affecting the coagulation strenght of different microbial rennets. Milchwiss., 25, 450.

[4] Houins (G.), Deroanne (Cl.), Coppens (R.) (1973). - Etude comparative de l'activité coagulante et du pouvoir protéolytique de la présure animale et de trois de ses succédanés. Le Lait, 529-530, 610.

[5] Ibrahim (M. K. E.), Amer (N. S.), El-Abol (M. M.) (1973). - Study on a microbial rennet produced by Mucor miehei. Egyptian J. Dairy Sci., 1, 127.

[6] Koning (P. J), Draaisma (J. Th. M.) (1973). - Identification of different types of rennet by means of isoelectric focusing. Neth. Milk Dairy J., 27, 368.

[7] Poznanski (S.), Reps (A.), Smietana (Z.) (1969). - Charakterystyka i prôby zastosowania ekstraktu pepsyny do koagulacji mleka przy wyrobie sera. Zesz. Nauk. WSR, Olsztyn, 25, 349.

[8] Prins (I.), Nelson (T. K.) (1974). - Microbial rennet Mucor miehei. Process. Biochem., 55, 34.

[9] Reps (A.), Poznanski (S.), Jakubowski (J.) (1975). - Main properties of milk coagulating preparations of microbial oxigin. Milchwiss., 30, 68. 